\title{
Magnetic resonance imaging and computed tomography features of alveolar soft-part sarcoma in the right deltoid muscle: A case report
}

\author{
HONGMEI LI ${ }^{*}$, JUN SUN* , JING YE and JINGTAO WU \\ Department of Radiology, Subei People's Hospital, Yangzhou University School of Medicine, \\ Yangzhou, Jiangsu 225001, P.R. China \\ Received January 11, 2015; Accepted February 8, 2016
}

DOI: $10.3892 / \mathrm{ol} .2016 .4290$

\begin{abstract}
Alveolar soft-part sarcoma (ASPS) is a rare, highly vascular malignant soft-tissue tumor that predominantly affects young adults. The tumor can occur in any bodily region, but is most frequently observed in the lower deep soft tissues of the extremities, and is rarely observed in the upper extremities. The present study reported a case of ASPS of the right deltoid muscle. The patient presented with a 3-year history of a mass on the right shoulder that exhibited rapid growth in the month prior to diagnosis. Computed tomography (CT) and magnetic resonance imaging (MRI) were performed. The lesion mainly demonstrated isointense or mildly hyperintense signals compared with the muscle on the T1-weighted images of the MRI, and heterogeneous high signal intensity on the T2-weighted images. CT enhancement showed a homogeneous enhanced mass. The tumor was resected and submitted for histopathological examination. The diagnosis was verified as ASPS by microscopic examination and immunohistochemical analysis. No distant metastases were noted. No evidence of local tumor recurrence was seen at 6 weeks following the wide surgical excision. The CT scan revealed no metastatic nodules in either lung during the follow-up. ASPS should be considered as a possible diagnosis when a slow-growing, large mass is detected in young adults in the soft tissue of the extremities, with high signal intensity and numerous signal voids on T1-weighted images (T1WI) and T2WI, and strong contrast-enhancement.
\end{abstract}

Correspondence to: Professor Jingtao $\mathrm{Wu}$, Department of Radiology, Subei People's Hospital, Yangzhou University School of Medicine, 98 Nantong West Road, Yangzhou, Jiangsu 225001, P.R. China

E-mail: drwujingtao@126.com

${ }^{*}$ Contributed equally

Abbreviations: ASPS, alveolar soft-part sarcoma; MRI, magnetic resonance imaging; T1WI, T1-weighted imaging; T2WI, T2-weighted imaging

Key words: alveolar soft-part sarcoma, surgery, sarcoma, magnetic resonance imaging

\section{Introduction}

Alveolar soft-part sarcoma (ASPS) is an infrequently encountered type of soft-tissue sarcoma that usually develops in the soft tissues of the extremities. The tumor accounts for $<1 \%$ of soft-tissue sarcomas and predominantly affects teenagers and young adults (1). ASPS has a high propensity for metastasis and the lungs are the most frequent site of metastasis by the hematogenous route. Although prolonged survival is possible even in patients with metastasis, the long-term disease specific mortality rate is high. The prognosis of ASPS is poor due to the high frequency of metastatic disease. A large study indicated that the median survival time was 3 years if metastatic disease was present at diagnosis and 11 years without metastatic disease at presentation $(1,2)$. ASPS is a rare, aggressive malignancy of uncertain histological origin, with a predisposition towards vascular invasion and distant metastasis. ASPS is most commonly observed in the extremities (1), but most commonly in the lower rather than the upper limbs. A previous study reported that ASPS is most commonly identified in the lower extremities (44\%) and is rarely seen in the upper extremities (2).

Magnetic resonance imaging (MRI) is the favored imaging modality for evaluation of this lesion due to its excellent soft tissue contrast, multiplanar imaging capability and lack of radiation exposure. Computed tomography (CT) enhancement and CT angiography clearly showed rich blood vessels within and around the tumor. The present study reports a case of ASPS of the right deltoid muscle, with emphasis on the clinical and unique imaging features that led to a correct diagnosis.

\section{Case report}

On April 22, 2014, a 30-year-old woman presented to Subei People's Hospital (Yangzhou, Jiangsu, China) with a mass on the right shoulder that had been apparent for 3 years, but that had rapidly grown in the last month. Physical examination revealed a well demarcated, soft, non-tender mass on the right shoulder. Sensory and motor examinations of the right upper limb were normal.

MRI and CT scanning of the right shoulder were performed using the 3.0-Tesla MRI scanner (Signa HDxt; 

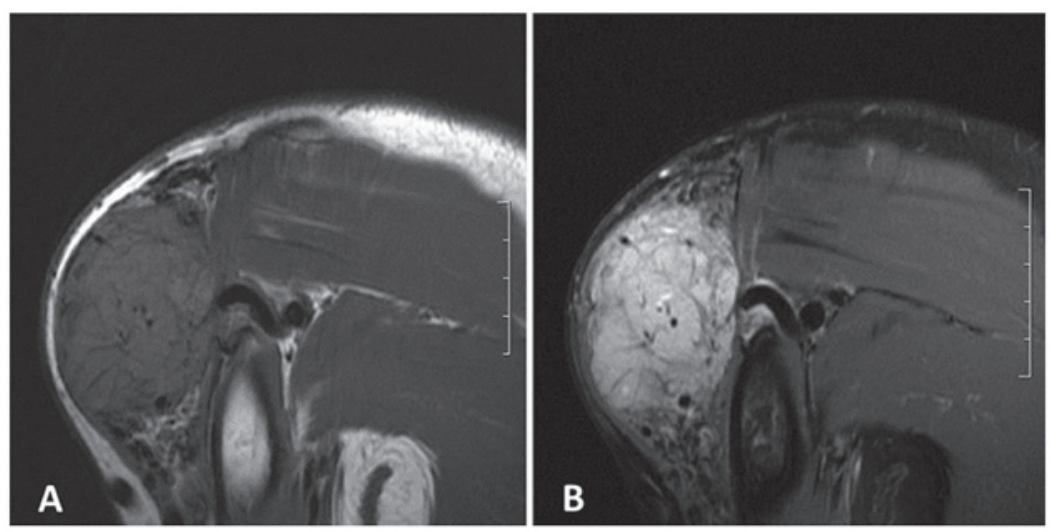

Figure 1. Magnetic resonance images of an alveolar soft-part sarcoma. (A) Coronal T1-weighted image. (B) Coronal T2-weighted fat-suppressed image. Slight hyperintensity was observed on the T1WI and hyperintensity was observed on the T2-weighted fat-suppressed image, with multiple signal voids.
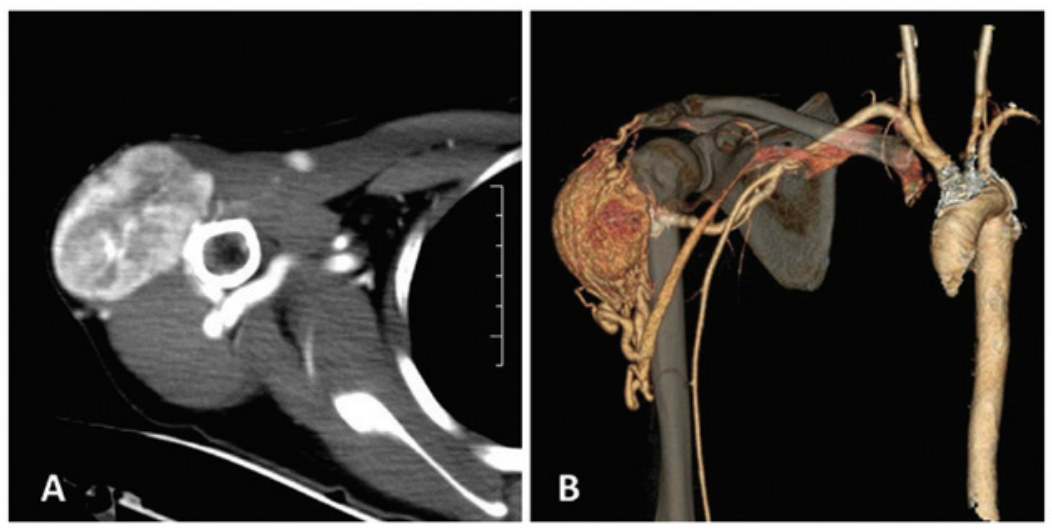

Figure 2. (A) Axial contrast-enhanced CT showing a relatively well-circumscribed mass with slightly greater peripheral enhancement. (B) CT angiography volume reconstruction showing a large hypervascular mass with multiple, enlarged tortuous vessels, and the subclavian artery and vein supplying blood to the tumor. CT, computed tomography.

GE Medical Systems, Milwaukee, WI, USA) and CT scanner (Discovery CT750 HD; GE Medical Systems). MRI revealed a hyperintense ovoid mass on the T2-weighted fat suppression image, and an isointense and hyperintense mass on the T1-weighted image (T1WI) compared with surrounding structures, with circuitous flow empty signals within and around the tumor (Fig. 1A and B). CT enhancement showed a homogeneous enhanced mass (Fig. 2A). CT angiography clearly showed rich blood vessels within and around a large hypervascular mass that was predominately supplied from the right subclavian artery, with venous drainage backflow into the subclavian vein (Fig. 2B). The bone structure of the right shoulder did not show any abnormalities. A surgical excision of the tumor was performed. During the procedure, the tumor mass was observed to be soft, hypervascular and well separated from the surrounding tissue. Tumor specimens were sent to the Department of Pathology, Subei People's Hospital, for histological and immunohistochemical analysis. The specimen was fixed using $10 \%$ formaldehyde, paraffin-embedded, stained with hematoxylin and eosin, dyed with periodic acid schiff and immunohistochemically stained for the detection of CD34. All antibodies and reagents are from Fuzhou Maixin Biotechnology Co., Ltd. (Fuzhou, China). A histological examination of the surgical specimens revealed nests of large granular cells separated

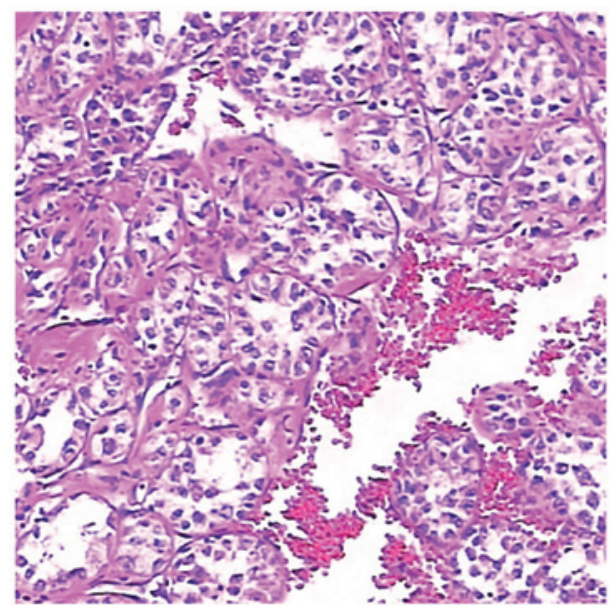

Figure 3. Histological findings showing a nest-like pattern of large, round to polygonal tumor cells with eosinophilic cytoplasm, exhibiting the classical alveolar pattern of growth (hematoxylin and eosin stain; magnification, $\mathrm{x} 100$ ).

by fibrovascular stroma. The cells were large and round to polygonal in shape, growing in the classical alveolar pattern (Fig. 3). Immunohistochemical findings showed that the cells were periodic-acid Schiff-positive. A final diagnosis of ASPS was made. 
At present, the patient remains alive. An MRI scan of the right shoulder showed no evidence of local tumor recurrence at 3,9 and 12 months following the wide surgical excision. A CT scan showed no metastatic nodules in either lung during the follow-up. The patient prognosis is good.

\section{Discussion}

ASPS is an extremely rare malignant tumor that constitutes $<1 \%$ of all soft-tissue sarcomas. The tumor exhibits a slight female preponderance in patients $\leq 30$ years old, in contrast to a male preponderance in patients $>30$ years old. The average age of onset falls within the second decade of life $(2,3)$.

In adults, ASPS is most commonly found in the lower extremities, followed by the trunk and upper extremities, whereas in infants and children, the majority of cases occur in the head and neck (3). The present study of a case in the upper extremity is relatively rare. The clinical course of the disease is slow, but with a high frequency of metastases, usually to the lungs, brain and bones. In the present study, the tumor occurred in a 30-year-old patient and the primary tumors were located in the extremity, representing a relatively indolent mass. These features are consistent with the previously reported literature, with the exception of a lack of metastasis.

ASPS derives its name from its histological appearance. Histologically, ASPS is characterized by a nest-like pattern of large, round to polygonal tumor cells with eosinophilic cytoplasm, exhibiting the classical alveolar growth pattern (4). These cells are PAS-positive with diastase-resistant intracytoplasmic inclusions. Immunohistochemical staining for CKpan, epithelial membrane antigen, MyoD1, myoglobin, myogenin, smooth muscle actin, desmin, CD34, CD10, Syn, chromogranin A and S-100 were negative (5). The present case was consistent with these histological features.

Regarding the imaging features, it has been noted that ASPSs mainly present as large and well-defined masses, with equal or slight hyperintensity on T1WI and hyperintensity on T2WI compared with the surrounding structures, and frequently occurring vascular signal voids (6). These features of a high signal intensity on T1WI and the presence of vascular signal voids within and around the tumor may represent the typical MRI features of ASPS (6). The precise cause of this high signal intensity on T1WI has not yet been elucidated. It has been hypothesized that this may be attributed to slow-flowing blood in or around the tumor and blood sinuses (cavities and separation between the blood vessels) in the tumor tissues. Vascular signal voids may be associated with rapid blood flow wash-out $(7,8)$. Non-contrast CT scans show the tumor to be of slightly high density and can display the location, scope, form and interior calcification of the tumor. However, they do not show the components in the tumor as well as MRI. Contrast-enhanced CT and MRI show intense, heterogeneous enhancement (9). Multi-slice spiral CT angiography can clearly display a hypervascular mass with multiple, enlarged tortuous vessels, and arteries and veins supplying blood to the tumor, which provides guidance for surgery (9). In the present case, the tumor exhibited a high signal intensity on T1WI and T2WI compared with the surrounding structures. The tumor was well enhanced on contrast-enhanced CT and MRI, and exhibited vascular signal voids. These reliable radiologic findings are well correlated with the high vascularity of ASPS in pathological specimens (10).

Several imaging features can assist in distinguishing ASPS from other benign or malignant tumors, such as hemangiomas and vessel malformations, synovial sarcoma and malignant fibrous histiocytoma (MFH) $(10,11)$. In ASPSs, vascular tissues surround the solid tumor tissues and blood flow wash-out is slow. This is in contrast to a high-flow vascular malformation, such as an arteriovenous malformation, with pure vascular tissue but no accompanying tissues, which enables the differentiation between the two conditions (12). The majority of sarcomas generally grow quickly, are relatively large and are located deep within the tissues, therefore, differentiating between them is occasionally difficult. However, certain imaging findings can assist in the diagnosis. Cases of synovial sarcoma typically occur in adults in and around the knee joint and lower leg, and tumor growth is slow and superficial, without flow voids or signal voids on MRI, but with calcification, necrosis and hemorrhage often noted on CT. Malignant fibrous histiocytoma is often observed in older men, and MRI showing necrosis, cystic regions and edema of the tumor is common while the blood vessels and nerves around the tumor are easily damaged. However, these features generally cannot be observed in ASPS, so recognition of these characteristic CT and MRI findings may lead to the early diagnosis of ASPS and avoid a misdiagnosis, which is important in the treatment of a small primary tumor (12).

According to the present study, when a slow-growing, painless superficial mass is encountered in the limbs, particularly the upper limbs, of young adult patients, the possibility of a sarcoma must be considered after a careful examination that eliminates all the benign possibilities, such as a ganglion or lipoma. The CT and MRI features of ASPS assist in providing a correct diagnosis and will aid surgeons in performing a wide surgical resection to reduce the risk of local recurrence. Angiography also adds useful diagnostic information. The ability to understand the imaging and clinical features of ASPS has certain value for the pre-operative qualitative diagnosis and clinical treatment of the tumor.

\section{References}

1. Van Vliet M, Kliffen M, Krestin GP and van Dijke CF: Soft tissue sarcomas at a glance: Clinical, histological, and MR imaging features of malignant extremity soft tissue tumors. Eur Radiol 19: 1499-1511, 2009.

2. Folpe AL and Deyrup AT: Alveolar soft-part sarcoma: A review and update. J Clin Pathol 59: 1127-1132, 2006.

3. Suh JS, Cho J, Lee SH, Shin KH, Yang WI, Lee JH, Cho JH, Suh KJ, Lee YJ and Ryu KN: Alveolar soft part sarcoma: MR and angiographic findings. Skeletal Radiol 29: 680-689, 2000.

4. Ladanyi M, Lui MY, Antonescu CR, Krause-Boehm A, Meindl A, Argani P, Healey JH, Ueda T, Yoshikawa H, Meloni-Ehrig A, et al: The der (17)t $(\mathrm{X} ; 17)(\mathrm{p} 11 ; \mathrm{q} 25)$ of human alveolar soft part sarcoma fuses the TFE3 transcription factor gene to ASPL, a novel gene at 17q25. Oncogene 20: 48-57, 2001

5. Argani P, Lal P, Hutchinson B, Lui MY, Reuter VE and Ladanyi M: Aberrant nuclear immunoreactivity for TFE3 in neoplasms with TFE3 gene fusions: A sensitive and specific immunohistochemical assay. Am J Surg Pathol 27: 750-761, 2003. 
6. Vilanova JC, Woertler K, Narváez JA, Barceló J, Martínez SJ, Villalón $\mathrm{M}$ and Miró J: Soft-tissue tumors update: MR imaging features according to the WHO classification. Eur Radiol 17: 125-138, 2007.

7. Lai YC, Chiou HJ, Wu HT, Chou YH, Wang HK and Chen PC: Ultrasonographic and MR findings of alveolar soft part sarcoma. J Chin Med Assoc 72: 336-339, 2009.

8. Iwamoto Y, Morimoto N, Chuman H, Shinohara N and Sugioka Y: The role of MR imaging in the diagnosis of alveolar soft part sarcoma: A report of 10 cases. Skeletal Radiol 24: 267-270, 1995

9. Kim HS, Lee HK, Weon YC and Kim HJ: Alveolar soft-part sarcoma of the head and neck: Clinical and imaging features in five cases. AJNR Am J Neuroradiol 26: 1331-1335, 2005.
10. Ogura K, Beppu Y, Chuman H, Yoshida A, Yamamoto N, Sumi M, Kawano $\mathrm{H}$ and Kawai A: Alveolar soft part sarcoma: A single-center 26-patient case series and review of the literature. Sarcoma 2012: 907179, 2012.

11. Dobson MJ, Hartley RW, Ashieigh R, Watson $\mathrm{Y}$ and Hawnaur JM: MR angiography and MR imaging of symptomatic vascular malformations. Clin Radiol 52: 595-602, 1997.

12. Pang LM, Roebuck DJ, Griffith JF, Kumta SM and Metreweli C: Alveolar soft-part sarcoma: A rare soft-tissue malignancy with distinctive clinical and radiological features. Pediatr Radiol 31: 196-199, 2001 